\title{
Intususcepción intestinal secundaria a tumor de Vanek
}

\author{
Intussusception secondary to Vanek's tumor
}

Pablo Merchán ${ }^{1}$

ORCID https://orcid.org/00000-0001-8283-4268

Enrique Petracchi ${ }^{2}$

ORCID https://orcid.org/0000-0002-2575-4656

Magali Muthular ${ }^{3}$

ORCID https://orcid.org/0000-0001-6934-095X

Lucía Rocco ${ }^{4}$

ORCID https://orcid.org/0000-0002-4215-0862

Carlos Canullán ${ }^{5}$

ORCID https://orcid.org/0000-0002-5755-0367

DOI 10.31837/cir.urug/5.2.4

Recibido: 5 de junio de 2020

Aceptado: 11 de abril de 2021

\section{Resumen}

El tumor de Vanek también conocido como pólipo fibroide inflamatorio es un tumor benigno de origen submucoso, que puede presentarse como un caso infrecuente de intususcepción en el adulto. Afecta a ambos sexos y es más frecuente entre la quinta y séptima década de vida. Presentamos el caso de una mujer de 42 años que consulta por dolor abdominal, con hallazgo de intususcepción secundaria a tumor de Vanek.

\footnotetext{
${ }^{1,4}$ Residente 3er año del servicio de Cirugía General del Hospital General de Agudos Dr. Cosme Argerich. Buenos Aires, Argentina.pmerchandelhierro@gmail.com ${ }^{2}$ Cirujano MAAC, Servicio de Cirugía General del Hospital General de Agudos Dr. Cosme Argerich. Buenos Aires, Argentina

${ }^{3}$ Residente 2do año del servicio de Cirugía General del Hospital General de Agudos Dr. Cosme Argerich. Buenos Aires, Argentina

${ }^{5}$ Cirujano MAAC y jefe de unidad del servicio de Cirugía General del Hospital General de Agudos Dr. Cosme Buenos Aires, Argentina
} 
Palabras Clave: Intususcepción; Pólipo fibroide inflamatorio; Tumor de Vanek

\begin{abstract}
Vanek's tumor also knows as inflammatory fibroid polyp is a benign tumor of submucosal origin, which can present as an infrequent case of intussusception in adults. It affects both sexes and is more frequent between the fifth and seventh decade of life. We present the case of a 42-year-old woman who consults for abdominal pain with a later diagnosis of intussusception due to tumor.
\end{abstract}

Key Words: Intussusception; Inflammatory fibroid polyp; Vanek’s tumor.

La intususcepción intestinal ocurre cuando una porción más proximal del intestino se invagina en el intestino distal. La patogenia se cree que implica una alteración en la peristalsis a causa de una lesión intraluminal, que es entonces un punto de referencia para la intususcepción.

La intususcepción en el adulto representa entre el 5 a 16\% de todos los casos de intususcepción y 1 a $5 \%$ de todos los casos de obstrucción intestinal. Esta intususcepción se debe en un $65 \%$ a neoplasias benignas o malignas, 15 a 25\% de los casos a patología no neoplásica, mientras que la intususcepción idiopática representa aproximadamente el $10 \%{ }^{(1)}$.

Presentamos el caso de una mujer de 42 años, sin antecedentes médicos, que consultó por dolor en flanco derecho de varias semanas de evolución. Niega náuseas, vómitos o alteración en el ritmo evacuatorio. En la tomografía computarizada de abdomen con contraste endovenoso se observó una imagen compatible con intususcepción en intestino delgado (fig. 1).

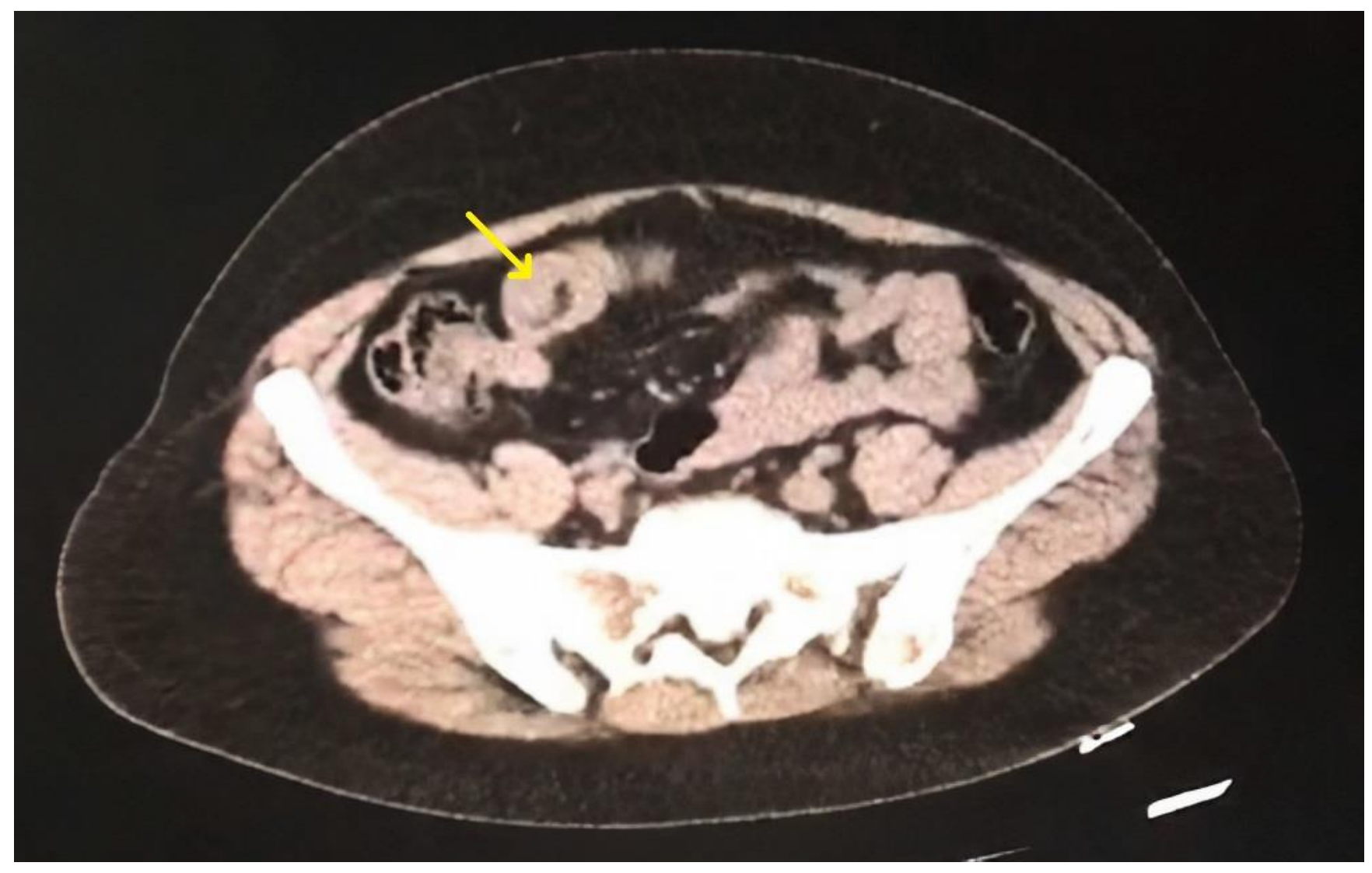


Fig. 1. Tomografía con contraste endovenoso. Invaginación intestinal por Tumor de Vanek (Flecha)

Se decidió realizar laparotomía exploradora donde se evidenció un tumor de íleon como etiología de la intususcepción; se realizó resección de intestino con entero-entero anastomosis. (fig. 2, fig. 3).

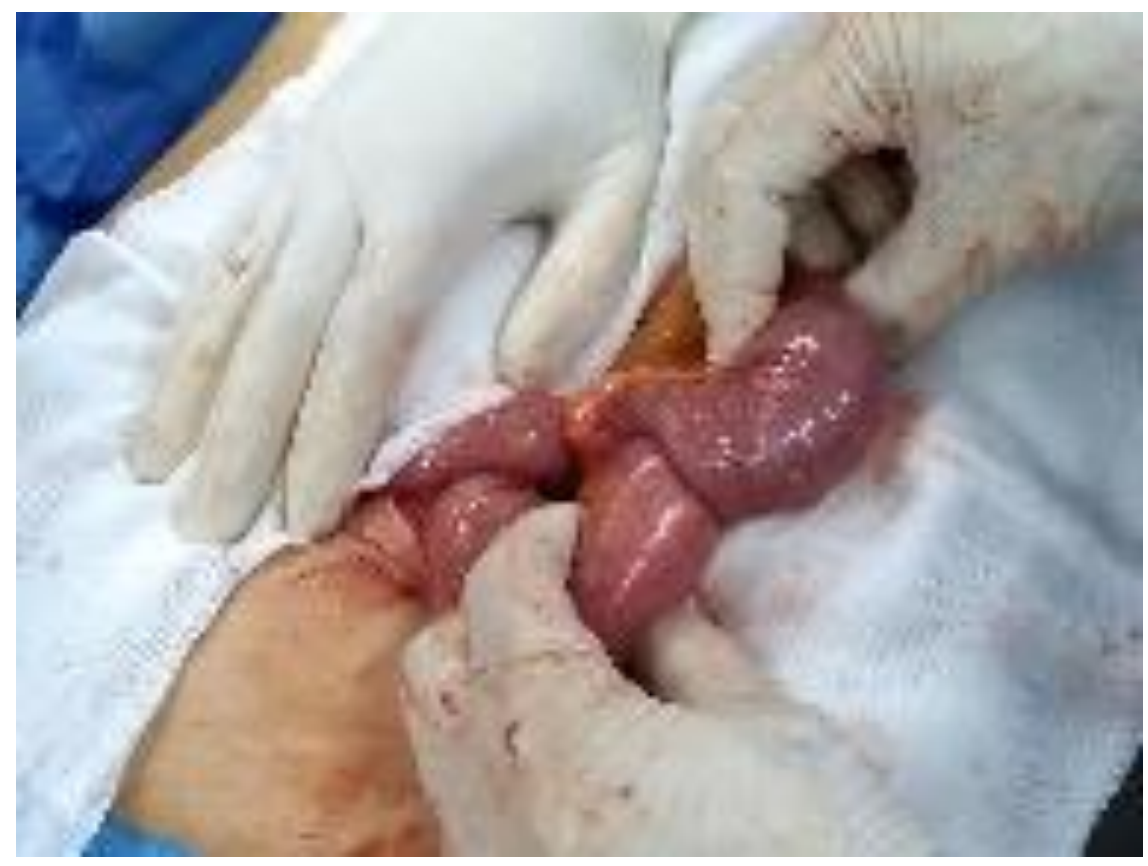

Fig. 2 Laparotomía exploradora. Invaginación intestinal

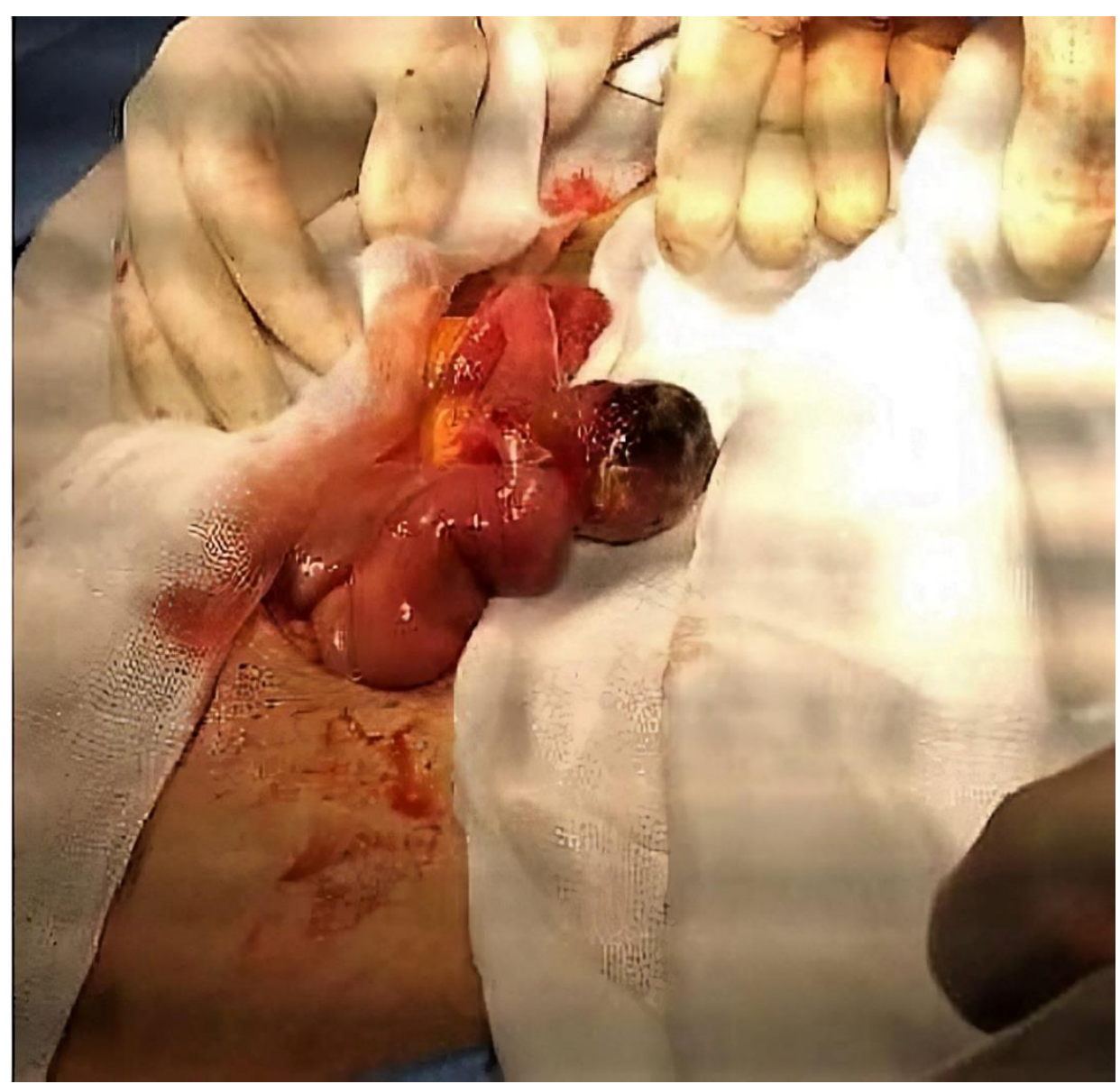


Fig. 3 Laparotomía exploradora. Enterotomía. Muestra pólipo fibroide inflamatorio (Tumor de Vanek)

La anatomía patológica informó un pólipo fibroide inflamatorio (PFI) también conocido como Tumor de Vanek.

Estos tumores son lesiones pseudotumorales idiopáticas infrecuentes del tracto gastrointestinal, que fueron descriptos por primera vez por Vanek en 1949 como un granuloma submucoso eosinofílico de naturaleza inflamatoria. (1) Afecta ambos sexos (con ligero predominio en hombres) y todos los grupos etarios, con un pico de incidencia entre la quinta y séptimas décadas de vida. ${ }^{(2)}$

La etiopatogenia de esta enfermedad es desconocida, se la vincula a una respuesta desproporcionada del huésped ante una injuria local con una importante proliferación del tejido conectivo a nivel de la submucosa de los diferentes órganos involucrados. ${ }^{(3)}$

El diagnóstico diferencial incluye el tumor de estroma gastrointestinal (GIST), leiomioma, schwannoma, perineuroma intestinal, y otros tumores benignos de la vaina nerviosa. ${ }^{(4)}$

Suelen ser asintomáticos y permanecen sin diagnosticarse durante mucho tiempo presentándose como hallazgos incidentales en la endoscopia y laparotomía. Cuando son sintomáticos, sus manifestaciones van a depender del tamaño y ubicación del tumor. El dolor abdominal es el síntoma principal en pacientes con lesiones en el estómago. Cuando el pólipo se encuentra en el intestino delgado se manifiesta como una oclusión o sub-oclusión intestinal debido a la intususcepción generada. Otros síntomas gastrointestinales, como vómitos, diarrea, heces con sangre, tenesmo y alteraciones en el hábito evacuatorio son menos frecuentes. ${ }^{2,5}$

La resección quirúrgica es el tratamiento de elección; es una herramienta diagnóstica y terapéutica para el tratamiento de esta enfermedad. ${ }^{(3)}$

La mayor parte de la información proviene de reportes de casos aislados. Un trabajo de seguimiento en una serie de 26 casos ha mostrado que tras su resección no requieren tratamiento adicional, ni presentan recurrencias. ${ }^{(5,6)}$

Ante un paciente con una oclusión intestinal secundaria a intususcepción de intestino delgado, el tumor de Vanek debe ser considerado entre sus diagnósticos diferenciales a pesar de su baja tasa de presentación. Su resolución es quirúrgica y el diagnóstico definitivo se realiza a través del examen anatomopatológico de la pieza quirúrgica. 


\section{Referencias}

1. Akbulut S. Intussusception due to inflammatory fibroid polyp : A case report and comprehensive literature review. World J Gastroenterol. 2012;18(40):5745-52.

2. Abboud B. Vanek' s tumor of the small bowel in adults. World J Gastroenterol 2015;21(16):4802-8.

3. Maya A, Gallo A, Castelli M, Paz L, Espinosa JC, Giunippero A. Intususcepción intestinal por pólipo fibroide inflamatorio en una Anciana. Medicina (Buenos Aires) 2013;461-3. Disponible en https://medicinabuenosaires.com/revistas/vol73-13/5/461-463MED5570_MAYA.pdf

4. Liu T C, Lin M, Montgomery E, Singhi A. Inflammatory Fibroid Polyps of the Gastrointestinal Tract Spectrum of Clinical, Morphologic, and Immunohistochemistry Features. Am. J Surg. Pathol. 2013;37(4):586-92. doi: 10.1097/PAS.0b013e31827ae11e

5. Correia Varela Almeida A, Cabello Fernández A, Brenda Minore N, Vázquez Morón JM, Gómez Delgado E, Bejarano García A. Tumor de Vanek: un hallazgo endoscópico poco común. RADP on line 2017;40:198-200. Disponible en. https://www.sapd.es/revista/2017/40/4/08

6. Acero D, Garijo G, Hombrados M, Figa M, Adrados M, Aldeguer X, et al. Pólipo fibroide inflamatorio gastrointestinal . Características clínicas y seguimiento de una serie de 26 pacientes. Gastroenterol Hepatol 2005;28(4):215-20. http://dx.doi.org/10.1157/13073089 